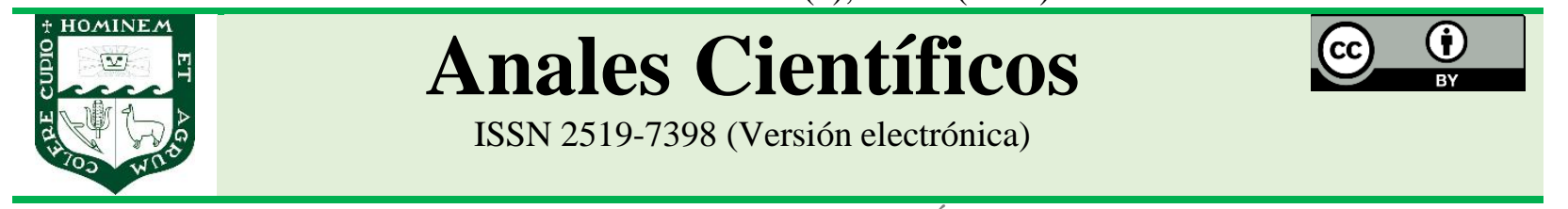

ARTÍCULO ORIGINAL - RESEARCH ARTICLE

http://dx.doi.org/10.21704/ac.v82i1.1742

\title{
PROPUESTA DE UN PLAN DE DESARROLLO DEL SECTOR FORESTAL PERUANO
}

\section{Proposal for a development plan for the Peruvian forestry sector}

\author{
Rafael Ramirez-Arroyo ${ }^{1 *(1)} ;$ Jesus Ramos Soto ${ }^{1}$ (i) \\ ${ }^{1}$ Facultad de Ciencias Forestales, Universidad Nacional Agraria La Molina, 15024, Lima, Perú. \\ * E-mail: rmramirez@lamolina.edu.pe
}

Recibido: 05/03/2020; Aceptado: 08/08/2021; Publicado: 31/08/2021

\begin{abstract}
The objective of this study was to develop a proposal for the National Forest and Wildlife Plan as a contribution to the mandate established in Law No. 29763. For this purpose, a qualitative descriptive method was used where a socio-economic, environmental diagnosis has been made and budgetary-institutional sector, to then be compared with global trends set by FAO. The main results indicate that, although an important group of entrepreneurs are increasing the production of products other than wood, those dedicated to wood production persist in producing goods that have had a clear decreasing trend worldwide. Another important aspect is the importance that environmental services have been acquiring with greater relevance, particularly those related to conservation and ecotourism, evidenced in the increase in natural protected areas, to the point that they currently occupy these 22 252077 ha. In relation to forestry institutions, this is very leafy and even though the public budget has increased in the last 11 years by 4.76 times, a better institutional, process and budget articulation is still necessary. In conclusion, to overcome these limitations, it is necessary that the forest development plan involves an adequate territorial ordering, an information system oriented to promotion, but also to the control of trade, a greater formal and real occupation of the territory, as well as the promotion of certified management areas, productive forest plantations and provision of environmental services, among other measures.
\end{abstract}

Keywords: forestry development; forest products; forestry legislation; forest policy

\section{RESUMEN}

El objetivo del presente estudio fue elaborar una propuesta para el Plan Nacional Forestal y de Fauna Silvestre como un aporte al mandato establecido en la Ley $\mathrm{N}^{\circ}$ 29763. Se utilizó un método descriptivo cualitativo donde se realizó un diagnóstico socio-económico, ambiental y presupuestal-institucional del sector, para luego ser comparado con las tendencias mundiales planteadas por FAO. Los principales resultados señalan que, si bien un grupo importante de empresarios están incrementando la producción de productos diferentes a la madera, aquellos dedicados a la producción maderera persisten en producir bienes que están con una clara tendencia decreciente a nivel mundial. Otro aspecto importante es la importancia que vienen adquiriendo con mayor relevancia los servicios ambientales, particularmente los vinculados a la conservación y ecoturismo, evidenciados en el incremento de áreas naturales protegidas, al punto que actualmente ocupan 22252077 ha. En relación con la institucionalidad forestal, es aún compleja y a pesar de que el presupuesto público se ha incrementado en los últimos 11 años en 4,76 veces, 
aún es necesario una mejor articulación institucional, de procesos y presupuestal. En conclusión, para superar estas limitaciones es necesario que el plan de desarrollo forestal involucre un adecuado ordenamiento territorial, un sistema de información orientado a la promoción, pero también al control del comercio; una mayor ocupación formal y real del territorio, así como la promoción de las áreas de manejo certificadas, de plantaciones forestales productivas y provisión de servicios ambientales, entre otras medidas.

Palabras clave: desarrollo forestal; productos forestales; legislación forestal; servicios ambientales; política forestal

Forma de citar el artículo (Formato APA):

Ramirez-Arroyo, R., \& Ramos, J. (2021). Propuesta de un plan de desarrollo del sector forestal peruano. Anales Científicos. 82(1), 63-72. http://dx.doi.org/10.21704/ac.v82i1.1742

Autor de correspondencia (*): Rafael Ramirez-Arroyo. Email: rmramirez@lamolina.edu.pe

(C) Los autores. Publicado por la Universidad Nacional Agraria La Molina.

This is an open access article under the CC BY

\section{INTRODUCCIÓN}

La superficie forestal del Perú abarca 68422585 hectáreas representando cerca del $60 \%$ de la superficie del territorio nacional (Ministerio del Ambiente, 2020). Estos recursos naturales han sido gestionados tradicionalmente desde una óptica que ha priorizado la extracción maderera enfocada en los bosques amazónicos, motivada por las facilidades legales para aprovechar la riqueza maderera existente.

La visión extractivista se vio plasmada en diversas normas legales sobre la temática forestal, como se aprecia en las diversas leyes forestales que se han emitido a lo largo de la historia Republicana; a saber la Ley del 21 de diciembre de 1898, la Ley $\mathrm{N}^{\circ} 1220$, Ley $\mathrm{N}^{\circ} 8928$, Ley $\mathrm{N}^{\circ} 10315$, Decreto Ley $\mathrm{N}^{\circ} 14552$, Decreto Ley $N^{\circ} 21147$, Ley $N^{\circ} 27308$ y Ley $N^{\circ} 29763$ (Congreso de la República, 2019a; Congreso de la República, 2019b; Congreso de la República, 2019c; Congreso de la República, 2019d; Congreso de la República, 2019e; Congreso de la República, 2019f; Congreso de la República, 2019g; Congreso de la República, 2019h; Congreso de la República, 2011).

Este enfoque no ha contribuido significativamente con la economía nacional ni tampoco ha eliminado la pobreza rural, tanto así que las estadísticas públicas difícilmente recogen información actualizada sobre el aporte del sector forestal a la economía nacional; como muestra de ello, el INEI (2008) citado en Ramírez (2010) señala que en el periodo 1994 - 2007 el aporte al PBI del sector agricultura, caza y silvicultura se hallaba alrededor del 6,5\%, en tanto que específicamente la sub actividad silvicultura aportaba alrededor del $0,0018 \%$ al PBI nacional.

En la Tabla 1, se señala que el potencial aprovechable de madera tan solo de la ecozona selva baja llega a los 8431492100 de metros cúbicos de madera, los cuales tomando como referencia la producción de madera rolliza nacional del año 2017, que fue de 1656206 de metros cúbicos de madera (SERFOR, 2019), debería permitir un abastecimiento creciente de madera casi a perpetuidad, contribuyendo con el desarrollo nacional.

Tabla 1. Volumen maderero por ecozonas (FAO y SERFOR, 2017).

\begin{tabular}{lccc}
\hline Ecozona & $\begin{array}{c}\text { Extensión } \\
\text { (Miles de ha) }\end{array}$ & $\begin{array}{c}\text { Volumen } \\
\left(\mathbf{m}^{\mathbf{3}} / \mathbf{h a}\right)\end{array}$ & $\begin{array}{c}\text { Volumen total } \\
\left(\mathbf{m}^{\mathbf{3}}\right)\end{array}$ \\
\hline Costa & 15025,082 & 2,87 & 43121985 \\
Sierra & 35263,940 & 5,68 & 200299170 \\
Selva Baja & 47142,813 & 178,85 & 8431492100 \\
Hidromórfica & 8669,706 & 113,29 & 982190990 \\
$\begin{array}{l}\text { Selva alta } \\
\text { accesible }\end{array}$ & 10942,030 & Sin datos & Sin datos \\
$\begin{array}{l}\text { Selva alta } \\
\text { difícil }\end{array}$ & 11477,988 & Sin datos & Sin datos \\
\hline Total & & & \\
\hline
\end{tabular}

Dentro de este contexto, el artículo $24^{\circ}$ de la vigente Ley Forestal y de Fauna Silvestre - Ley No 29763 dispone que "la planificación forestal y de fauna silvestre se enmarca en la política nacional forestal y de fauna silvestre" (Congreso de la República, 2011), la cual fue aprobada mediante Decreto Supremo No 009-2013MINAGRI (MINAGRI, 2013), estando estructurada de 
la siguiente manera: Eje Institucionalidad y gobernanza (conteniendo dos lineamientos y dieciséis declaraciones), sostenibilidad (que contiene dos lineamientos y dieciséis declaraciones), competitividad (con un lineamiento y catorce declaraciones), inclusión social e interculturalidad (conteniendo dos lineamientos y doce declaraciones) y conocimientos, ciencia y tecnología (con dos lineamientos y ocho declaraciones) (MINAGRI, 2013).

Toda vez que las políticas públicas son declaraciones que priorizan objetivos a desarrollar por el Estado, a las que deben asignárseles recursos limitados, la actual Política Nacional Forestal y de Fauna tiene la característica de ser muy amplia, y por ello la propia Ley Forestal y de Fauna Silvestre señala la necesidad de establecer un Plan Nacional Forestal y de Fauna Silvestre donde se entiende deben priorizarse las acciones del Estado para el desarrollo del sector.

Un principio general de la Ley $\mathrm{N}^{\circ} 29763$ es la gobernanza forestal y de fauna silvestre (Congreso de la República, 2011), razón por la cual el presente trabajo tiene como objetivo central, elaborar y proponer una propuesta para el Plan Nacional Forestal y de Fauna Silvestre para lograr que los recursos forestales, de fauna silvestre y servicios ambientales del Perú sean una fuente efectiva a favor del desarrollo sostenible nacional y contribuyan a reducir la pobreza principalmente en el ámbito rural.

\section{MATERIALES Y MÉTODOS}

El presente estudio utilizó un método descriptivo cualitativo; para ello se realizó una recopilación, selección y sistematización de datos e información objetiva acerca del estado situacional del sector forestal y de fauna silvestre peruano. A fin de contar con una visión holística del sector, los datos e información recopilados fueron clasificada en tres grandes grupos:

- Información socio-económica: Vinculada al comercio de los productos provenientes de los recursos forestales y de fauna silvestre.

- Información ambiental: Que involucró el estado en cuanto a las distintas figuras legales vigentes en nuestra legislación destinadas a ocupar el territorio forestal, llámense áreas naturales protegidas, títulos habilitantes, actos administrativos que no son títulos habilitantes, entre otros.

\section{- Información presupuestal-institucional}

Relacionada al marco institucional y presupuesto asignado por el Congreso a entidades públicas que tienen competencias en el sector.

Se tomaron como referencia las tendencias internacionales sobre oferta, demanda y corrientes que influyen en el desarrollo del sector, planteadas en el estudio titulado: Tendencias y perspectivas del sector forestal en América latina y el Caribe (FAO, 2006) cuyo resumen se aprecia en la Tabla 2, para luego efectuar una comparación con el estado situacional hallado.

Tabla 2. Tendencias del sector forestal y de fauna silvestre para Latinoamérica por grupo de recursos o productos (FAO, 2006).

\section{Bosques naturales, plantaciones y madera}

- La demanda de productos forestales está creciendo mundialmente en un 15\% (estimado entre el 2005 al 2020) y está formada principalmente por pulpa, papel, productos de madera sólida (cada vez con mayor valor agregado) y tableros aglomerados.

- La reducción de áreas de bosques manejados generará que su producción se reduzca en promedio: $1,8^{\prime}$ metros cúbicos/año.

- Las exigencias de los países consumidores desarrollados harán necesaria una mayor certificación de bosques naturales manejados, así como la estandarización de sus productos.

- Se estima el aumento del área de plantaciones forestales de 13,1 ' ha (2005) a 17,3' ha (2020) en la región.

Tableros de diversos tipos y similares

- La producción de tableros de madera en América Latina y el Caribe creció $144 \%$ en 20 años, siendo sustituto de la madera sólida.

- Otros sustitutos de la madera sólida son: el policloruro de vinilo (PVC) y el aluminio (para ventanas, puertas y costaneros), el vidrio (para puertas y muebles), la cerámica usada en azulejos o el vinilo (para pisos), el concreto (para construcción estructural, postes de transmisión), el plástico en laminados (para las chapas, para ventanas, puertas y muebles), el hormigón (para durmientes de ferrocarril) y el acero (para las grandes construcciones). Entre los productos sustitutos: tableros de yeso (para divisiones, revestimientos de paredes), tablero de fibra de cemento (para cielo raso, revestimientos de paredes), ladrillos (para construcción), cables estructurales, vigas, soportes, muebles.

- Sin embargo, aquella madera proveniente de una producción sostenible cuenta con una demanda importante. 


\begin{tabular}{l}
\hline Productos diferentes a la madera \\
\hline - El comercio de productos diferentes a la madera tiene \\
una tendencia constante a mejorar en todos sus usos, \\
tales como extractivos del tronco, resinas de pino (Pinus \\
spp.), gomas; aceites esenciales como del eucalipto \\
(Eucalyptus spp.); caucho natural de Hevea brasiliensis; \\
colorantes de achiote (Bixa orellana); alimentos como la \\
castaña de Pará (Bertholletia excelsa), palmito (Euterpe \\
spp., Bactris Gasipaes y Guilielma spp.), bebidas como \\
el guaraná (Paullinea cupana), yerba mate (Ilex \\
paraguariensis), etc; plantas medicinales y material para \\
la construcción civil, fabricación de muebles y artesanía \\
como el bambú, caña de Guayaquil, carrizo, etc. \\
\hline Servicios ecosistémicos \\
- El aumento de la conciencia ambiental y los acuerdos \\
internacionales mantendrá la tendencia de conformación \\
de áreas naturales protegidas y otras áreas para \\
conservación y servicios ambientales públicas y \\
privadas para diversos usos, tales como recreación, \\
ecoturismo, conservación del ciclo hidrológico, etc. \\
\hline
\end{tabular}

Finalmente, producto de la contrastación se propusieron los aportes de políticas al plan de desarrollo del sector forestal peruano.

\section{RESULTADOS Y DISCUSIÓN}

En las Tablas 3, 4 y 5 se presenta información sobre el desenvolvimiento comercial de los productos forestales y de fauna silvestre, correspondiente a los hitos desde el año 1983 al 2017 referida a producción, exportación e importación de estos productos, también se aprecia el comportamiento de la producción y comercio en este sector. La producción maderera por tipo de productos no presenta una tendencia creciente, por el contrario, históricamente presenta una tendencia decreciente, debido a la persistencia en la industria nacional en producir bienes no acordes a las tendencias mundiales vinculadas a los productos forestales. Asimismo, se complementa con el hecho de que la balanza comercial en cuanto a productos maderables ha sido históricamente negativa (con la excepción del año 1993), apreciándose que la proporción entre los valores de importación y exportación se halla entre $2: 1$ y $3: 1$ aproximadamente. La demanda nacional no se abastece con los productos nacionales, sea por el tipo de producto, su calidad u otros factores y requiere de productos de la industria extranjera.

Para revertir esta situación es fundamental que el Estado realice una labor de inteligencia comercial, genere información relevante que promueva la actividad y ofrezca nuevas oportunidades a los empresarios, promoviendo si es necesario y temporalmente las exportaciones maderables en diferentes niveles de procesamiento según las demandas mundiales y promoviendo en el caso de madera obtenida de bosques bajo manejo la certificación forestal.

Para ello, sin que se descuide la lucha contra la tala ilegal controlando el transporte y comercio de productos forestales, el sistema de información forestal y de fauna silvestre debe priorizar la promoción del sector.

Es de resaltar que para el caso de los productos diferentes a la madera, la tendencia de exportación ha sido constantemente creciente, siendo el caso que las exportaciones recientes equivalen a más de 38 veces a las registradas en 1983, hecho positivo que es coherente con las tendencias mundiales y que debe fortalecerse promoviendo un mayor valor agregado y volúmenes involucrando a las comunidades nativas y campesinas, las cuales tienen el conocimiento ancestral sobre la identificación y uso sobre estos productos. Es necesario indicar que, así como las exportaciones se incrementan, ello trae consigo un aumento en el riesgo de fuga de recursos genéticos, por ello debe existir mayor control sobre este tema.

Especial atención merece la agroforestería con café y cacao, actividad que puede aportar a la mejora de ingresos en las zonas rurales y donde la legislación ofrece alternativas para ello.

En relación con otros usos de los bosques, en la Tabla 6 se presenta una aproximación al avance en cuanto al área gestionada por el Estado y el sector privado, siendo el mayor avance la implementación de áreas naturales protegidas tanto nacionales como de conservación regional y privadas (estas últimas en constante aumento). Es de resaltar que estas áreas se establecen entre otros fines, para desarrollar ecoturismo y en general servicios ambientales, actividades compatibles con la legislación forestal y de fauna silvestre y que por tanto pueden también ser promovidas por las autoridades nacionales y regionales forestales.

De la Tabla 6 se desprende también que nominalmente, el $34 \%$ del territorio de aptitud forestal y de protección 
tiene algún tipo de ocupación por título habilitante o norma legal, pudiendo ser el porcentaje efectivo mucho menor; ello conduce a considerar necesaria la ocupación formal del bosque, para lo cual los procesos de zonificación, ordenamiento, titulación de comunidades nativas y campesinas, catastro rural y el desarrollo de inventarios de recursos forestales y de fauna silvestre en sus diversos niveles deben impulsarse.

La superficie reforestada, se halla alrededor del 10\% del potencial teórico; en términos relativos es bajo, pero entendiendo la alta productividad por la que debería tener una plantación forestal frente a un bosque manejado por ha, advertimos que las plantaciones nacionales no están constituyendo fuentes de ingresos, sea porque las estadísticas públicas presentan errores, sea porque son plantaciones con fines diferentes a los de producción, sea porque no se definió bien el objeto de la plantación, por ser sobre maduras u otras razones; por ello, es necesario promover e implementar con capitales públicos y privados plantaciones productivas donde no solo estén involucradas las tierras de capacidad mayor forestal que per se no pueden ser propiedad privada o implican trabajar directamente con comunidades campesinas; ante esto, una alternativa es trabajar en aquellas tierras para pastos o agricultura permanente de menor calidad, donde existe propiedad privada y donde es legal y puede ser económicamente viable la plantación y manejo productivo.

Tabla 3. Evolución histórica reciente de la producción forestal maderable (En metros cúbicos) (SERFOR, 2019; SERFOR, 2018; SERFOR, 2016; MINAGRI, 2015; MINAGRI, 2014; INRENA,2003; INRENA, 1997).

\begin{tabular}{|c|c|c|c|c|c|c|c|c|}
\hline Producto/Año & 1983 & 1993 & 2003 & 2013 & 2014 & 2015 & 2016 & 2107 \\
\hline Madera rolliza & 4111300 & 8083300 & 1384510 & 2013559 & 2044028 & 1694431 & 1448367 & 1656206 \\
\hline $\begin{array}{l}\text { Madera laminada y } \\
\text { chapas decorativas }\end{array}$ & 35100 & 13800 & 9591 & 6967 & 4297 & 4989 & 1933 & 4942 \\
\hline Triplay & 50400 & 82600 & 101326 & 64824 & 46413 & 50960 & 49592 & 53290 \\
\hline Parquet & 12000 & 18300 & 7341 & 14410 & 19552 & 10190 & 5834 & 12869 \\
\hline Postes & 4300 & 1200 & 8055 & 255 & 109 & 7 & 0 & 0 \\
\hline Durmientes & 5200 & 7600 & 3081 & 4761 & 2938 & 665 & 623 & 880 \\
\hline Madera aserrada & 729300 & 1123600 & 528292 & 575262 & 667153 & 579079 & 333266 & 482328 \\
\hline Carbón $(\mathrm{kg})^{*}$ & 21400000 & 4050000 & 18146634 & 46605510 & 59064999 & 20347985 & 4547029 & 29275512 \\
\hline Leña & 3232400 & 6828200 & 7243180 & 7028267 & 7028267 & 7028267 & 7028267 & 7028267 \\
\hline
\end{tabular}

(*) Un $\mathrm{m}^{3}$ de carbón $=500 \mathrm{~kg}$

Tabla 4. Evolución histórica reciente de las importaciones de productos forestales maderables y diferentes a la madera (Valor CIF en miles de U\$) (SERFOR, 2019; SERFOR, 2018; SERFOR, 2016; MINAGRI, 2015; MINAGRI, 2014; INRENA,2003; INRENA, 1997).

\begin{tabular}{lcrrrrrrr}
\hline \multicolumn{1}{c}{ Producto } & $\mathbf{1 9 8 3}$ & $\mathbf{1 9 9 3}$ & \multicolumn{1}{c}{$\mathbf{2 0 0 3}$} & $\mathbf{2 0 1 3}$ & $\mathbf{2 0 1 4}$ & $\mathbf{2 0 1 5}$ & \multicolumn{1}{c}{$\mathbf{2 0 1 6}$} & \multicolumn{2}{c}{$\mathbf{2 1 0 7}$} \\
\hline Productos maderables & 4195,00 & 5932,00 & 270480,86 & 1063166,77 & 1116575,51 & 1079834,86 & 1025401,47 & 962147,14 \\
Productos diferentes & 4438,35 & 11787,93 & 17554,52 & 77023,40 & 80047,11 & 63052,62 & 63830,82 & 72845,00 \\
a la madera & 8633,35 & 17719,93 & 288035,37 & 1140190,18 & 1196622,62 & 1142887,48 & 1089232,29 & 1034991,92 \\
Total & & & & &
\end{tabular}


Tabla 5. Evolución histórica reciente de las exportaciones de productos forestales y de fauna silvestre (Valor FOB en miles de U\$) (SERFOR, 2019; SERFOR, 2018; SERFOR, 2016; MINAGRI, 2015; MINAGRI, 2014; INRENA,2003; INRENA, 1997).

\begin{tabular}{|c|c|c|c|c|c|c|c|c|}
\hline Producto /Año & 1983 & 1993 & 2003 & 2013 & 2014 & 2015 & 2016 & 2107 \\
\hline Productos maderables & 3651311 & 14997619 & 122924134 & 252574148 & 264458758 & 214854275 & 206754960 & 120696892 \\
\hline $\begin{array}{l}\text { Productos diferentes a la } \\
\text { madera }\end{array}$ & 4711790 & 11467905 & 32723697 & 137719884 & 134311762 & 149169133 & 177651062 & $\begin{array}{r}181041874 \\
\text { (1) }\end{array}$ \\
\hline $\begin{array}{l}\text { Fauna silvestre sin fibra } \\
\text { de vicuña }\end{array}$ & Sin datos & Sin datos & Sin datos & Sin datos & Sin datos & Sin datos & 8638850 & 6342949 \\
\hline Fibra de vicuña & Sin datos & Sin datos & Sin datos & Sin datos & Sin datos & Sin datos & 4084561 & 2299762 \\
\hline Total & 8363101 & 26465524 & 155647831 & 390294033 & 398770520 & 364023408 & 397129433 & 310381477 \\
\hline
\end{tabular}

Tabla 6. Tierras con cobertura forestal gestionadas por el Estado y el sector privado (Cortez, 2015; SERFOR, 2019; SERNANP, 2020; MINAM, 2020).

\begin{tabular}{lcccccc}
\hline Capacidad de uso mayor & Potencial (ha.) & $\begin{array}{c}\text { Superficie } \\
\text { Reforestada }\end{array}$ & $\begin{array}{c}\text { Mecanismo } \\
\text { Transferencias directas } \\
\text { condicionadas }\end{array}$ & $\begin{array}{c}\text { Títulos } \\
\text { habilitantes }\end{array}$ & $\begin{array}{c}\text { Áreas } \\
\text { naturales } \\
\text { protegidas }\end{array}$ & $\begin{array}{c}\text { Porcentaje } \\
\text { autorizado o } \\
\text { implementado }\end{array}$ \\
\hline $\begin{array}{l}\text { Forestal (aptas para } \\
\text { reforestación) }\end{array}$ & 10500000 & 1069768 & -- & -- & -- & 10,18 \\
$\begin{array}{l}\text { Forestal(restante) y } \\
\text { Protección }\end{array}$ & 92496560 & -- & 1955283 & 9802636 & 22252077 & 36,77 \\
Total & 102996560 & 1069768 & 1955283 & 9802636 & 22252077 & 34,08 \\
\hline
\end{tabular}

Tabla 7. Presupuesto institucional modificado correspondiente al año 2019 de las principales instituciones públicas vinculadas al sector forestal y de fauna silvestre (MEF, 2020).

\begin{tabular}{|c|c|c|c|}
\hline Sector & $\begin{array}{r}\text { Presup } \\
\text { PI } \\
\end{array}$ & $\begin{array}{l}\text { Ipuesto inicial modificado- } \\
\text { IM (Millones de Soles) }\end{array}$ & $\begin{array}{c}\text { Porcentaje del PIM } \\
\text { total asignado }\end{array}$ \\
\hline \multicolumn{4}{|c|}{ Ministerio del Ambiente } \\
\hline & SERNANP & 95369952 & 25,13 \\
\hline & Programa Bosques & 36387073 & 9,59 \\
\hline \multicolumn{4}{|c|}{ Ministerio de Agricultura y } \\
\hline Riego & SERFOR & 105435088 & 27,79 \\
\hline \multicolumn{4}{|c|}{ Presidencia del Consejo de } \\
\hline Ministros & OSINFOR & 39194158 & 10,33 \\
\hline \multicolumn{4}{|l|}{ Gobiernos Regionales } \\
\hline & Programas u oficinas & & \\
\hline & $\begin{array}{l}\text { vinculadas a la gestión forestal } \\
\text { y de fauna silvestre }\end{array}$ & 103015807 & 27,16 \\
\hline Total & & 379402020 & 100,00 \\
\hline
\end{tabular}


Tabla 8. Propuesta de Plan Nacional Forestal y de Fauna Silvestre.

\section{Lineamientos de políticas públicas}

1. El Perú debe completar la zonificación forestal, el ordenamiento territorial y los inventarios de recursos forestales y de fauna silvestre.

2. El país debe contar con un catastro nacional de tierras forestales debidamente actualizado a fin de dar seguridad jurídica a las inversiones forestales.

3. Las áreas de manejo forestal maderero otorgadas en concesiones y permisos en bosques tropicales deben estar certificadas.

4. El Estado debe promover la ocupación legal del bosque, priorizando la titulación de las comunidades nativas y campesinas y estudiar la viabilidad de otorgar propiedad privada en tierras de aptitud forestal degradadas modificando la legislación pertinente.

5. El sector privado debe ofrecer una mejor calidad, volúmenes y valor agregado a sus productos diferentes a la madera; en tanto que los centros de investigación deben identificar los productos diferentes a la madera y componentes activos de estos priorizando el trabajo con las comunidades nativas y campesinas.

6. El sector público, el sector privado y la inversión mixta deben establecer plantaciones forestales en tierras de capacidad de uso mayor C3 y P3 con propiedad privada, así como en tierras de capacidad de uso mayor F cercanas a centros de consumo local y a través de compras públicas que promuevan el desarrollo de empresas forestales formales, otorgando beneficios tributarios y de otra índole.

7. El Estado debe incluir dentro de sus políticas para el desarrollo del sector forestal, aquellas encaminadas a mejorar las condiciones de educación, sanidad, transporte, combate a la pobreza y lucha contra el narcotráfico en zonas rurales.

8. El Estado debe promover por un tiempo determinado la exportación de productos demandados en el mercado exterior al margen de su nivel de transformación, a fin de generar ingresos a las unidades económicas.

9. Debe fortalecerse la institucionalidad pública (normatividad, recursos, institucionalidad, etc.) que controle el acceso a los recursos genéticos.

10. Deben transferirse las funciones forestales a los gobiernos regionales de manera progresiva y fortaleciendo sus capacidades, procurando que el SERFOR asuma un claro liderazgo técnico.

11. El SERFOR debe promover el otorgamiento de títulos habilitantes con el fin de ofrecer servicios ambientales priorizando el ecoturismo y los servicios ambientales tales como la conservación del agua.

12. Debe articularse la institucionalidad forestal no solo a través de los sistemas funcionales señalados en la Ley 29763 sino también a través de programas presupuestales interinstitucionales.

13. Se debe construir un sólido sistema nacional de información forestal y de fauna silvestre destinado a promover el sector y también para combatir la tala ilegal de madera y el comercio ilícito de productos diferentes a la madera y fauna silvestre.

14. Promover el desarrollo de la agroforestería con café y cacao en base a la legislación vigente.

En cuanto a la institucionalidad forestal, existen en la actualidad diversas entidades involucradas en la temática forestal, a nivel regulatorio, de gestión y promoción confluyen el SERFOR y SERNANP conjuntamente con los diversos gobiernos regionales donde se han transferido funciones forestales; en tanto que a nivel del control y fiscalización las entidades involucradas son más numerosas aún, ya que incluyen además de las ya indicadas al OSINFOR, la Policía Nacional del Perú, a las Fuerzas Armadas, Ministerio Público, Poder Judicial, SUNAT, entre otras. Otras actividades como la investigación forestal se hallan más fraccionada por involucrar entidades como el CONCYTEC, INIA, PRODUCE, universidades, entre otras; lo que refleja la alta complejidad en la gestión integral de los recursos forestales y de fauna silvestre.

Por otra parte, a nivel presupuestal, si se toma como referencia el año 2008, en el cual aún no operaba el Ministerio del Ambiente, no se habían ejecutado transferencia de funciones a los gobiernos regionales y la gestión de los recursos forestales y de fauna silvestre se hallaba centralizada en el extinto Instituto Nacional de Recursos Naturales, la institucionalidad forestal tuvo como presupuesto inicial modificado S/ 79686627 (MEF, 2020), a diferencia de lo asignado en el año 2019, que puede apreciarse en la Tabla 7 y que ascendió a S/ 379402 
020. Este presupuesto equivale 4,76 veces el aprobado para el año 2008, refleja que los recursos económicos se han incrementado ostensiblemente acorde a la alta complejidad institucionalidad; sin embargo, se aprecia que las autoridades que se hallan más próximas a la gestión del territorio reciben en conjunto, poco más de la cuarta parte de todo presupuesto; lo que debe hacer reflexionar sobre una inequidad en la asignación de fondos públicos, además de una necesidad imperiosa de coordinación interinstitucional, de avanzar en la transferencia de funciones a los gobiernos regionales acompañado de recursos, que respondan a una articulación presupuestaria a través de los presupuestos por resultados, con lo que se estaría empoderando a los gobiernos regionales con acompañamiento de un claro liderazgo articulador del SERFOR.

Aunado a lo anterior, por la lejanía y difícil acceso a estos recursos naturales, es fundamental que el desarrollo forestal esté acompañado de mejores condiciones para el poblador rural, vinculadas estas a sanidad, comunicaciones, entre otras que articuladas permitan un desarrollo adecuado.

Finalmente, y luego de una evaluación de las tendencias mundiales sobre el manejo y comercio de productos forestales, así como el estado actual de la actividad forestal; el estudio arroja catorce lineamientos de políticas presentados en la Tabla 8, que constituyen un aporte para el desarrollo del sector forestal a un mediano plazo y que deberían ser la base para el desarrollo del Plan Nacional Forestal y de Fauna Silvestre.

\section{CONCLUSIONES}

La búsqueda del progreso del sector forestal peruano debe descartar aquel enfoque donde se prioriza únicamente el potencial forestal y de fauna silvestre con que el país cuenta, entendido este, como la oferta de recursos; el estudio demuestra que por el contrario, en un mundo globalizado, donde muchos países con claras ventajas frente al Perú, compiten por conservar o aumentar mercados, nuestro país debe enfocar sus instrumentos de planificación públicos, tales como la Política Nacional Forestal y de Fauna Silvestre y el Plan de Desarrollo Forestal en analizar la demanda mundial de recursos, productos primarios $\mathrm{y}$ productos secundarios $\mathrm{y}$ trabajar en satisfacerla, adecuándose como país a las tendencias mundiales, teniendo claro que el motor que impulse el futuro del sector forestal debe ser el establecimiento y aprovechamiento de plantaciones forestales. Por tanto, ante la actual falta de esta prioridad, se hace imperativo revisar y aprobar una nueva Política Nacional Forestal y de Fauna Silvestre, su respectivo Plan de Desarrollo y, seguidamente hacer cambios normativos imprescindibles, tales como permitir la propiedad privada en tierras de aptitud forestal degradadas y revisar la compleja y desarticulada institucionalidad forestal actual. A ello debe unirse un agresivo plan de promoción y facilitación para que el sector privado, con la participación de sus diversos actores, incluidas las MYPES, medianas y grandes empresas inviertan con confianza en este sector.

\section{Conflictos de intereses}

Los autores firmantes del presente trabajo de investigación declaran no tener ningún potencial conflicto de interés personal o económico con otras personas $\mathrm{u}$ organizaciones que puedan influir indebidamente con el presente manuscrito.

\section{Contribuciones de los autores}

Preparación y ejecución: RRA, JRS; Desarrollo de la metodología: RRA, JRS; Concepción y diseño: RRA, JRS; Edición del artículo: RRA, JRS; Supervisión del estudio: RRA, JRS.

\section{LITERATURA CITADA}

- Congreso de la República. (2011). Ley Forestal y de Fauna Silvestre. Ley No 29763. En: Diario oficial El Peruano: Lima 22 de julio del 2011. Año XXVIII - No 11475, pp: 446980 -447004

- Congreso de la República. (2019 $)$. Estableciendo mecanismos para la colonización de las montañas. Ley s/n del 21 de diciembre de 1898. Disponible en http://www.leyes.congreso.gob.pe/

- Congreso de la República. (2019b). Legislación sobre terrenos de montaña. Ley $N^{\circ} 1220$ del 21 de diciembre de 1909. Disponible en 
https://www.leyes.congreso.gob.pe/Documento s/Leyes/01220.pdf

- Congreso de la República. (2019c). Disponiendo que las maderas en troncos o aserradas, provenientes de los bosques del territorio nacional y que se destinen a los centros de consumo utilizando las autovías de penetración de montaña, pagarán el mismo impuesto de un centavo por pie cuadrado. Ley No 8928 del 20 de julio del 1939. Disponible en https://www.leyes.congreso.gob.pe/Documento s/Leyes/08928.pdf

- Congreso de la República. (2019d). Disponiendo que todos los colonos del Perú puedan explotar y comerciar libremente la madera proveniente de los bosques que colonicen. Ley $\mathrm{N}^{\mathrm{o}} 10315$ del 15 de diciembre de 1945. Disponible en https://www.leyes.congreso.gob.pe/Documento s/Leyes/10315.pdf

- Congreso de la República. (2019e). Creando el Servicio Forestal y de Caza como organismo de derecho público interno, anexo al Ministerio de Agricultura. Decreto Ley $N^{o} 14552$ del 11 de julio de 1963. Disponible en de https://www.leyes.congreso.gob.pe/Documento s/Leyes/14552.pdf

- Congreso de la República. (2019f). Ley forestal y de fauna silvestre. Decreto Ley No 21147 del 13 de mayo de 1975. Disponible en https://www.leyes.congreso.gob.pe/Documento s/Leyes/21147.pdf

- Congreso de la República. (2019g). Ley forestal y de fauna silvestre. Decreto Ley No 21147 del 13 de mayo de 1975. Disponible en https://www.leyes.congreso.gob.pe/Documento s/Leyes/27308.pdf

- Congreso de la República. (2019h). Ley forestal y de fauna silvestre. Ley $N^{\circ} 27308$ del 07 de julio del 2000. Disponible en https://www.leyes.congreso.gob.pe/Documento s/Leyes/27308.pdf

- Cortez, A. (2015). Marco teórico relieve-suelotierra. 29pp Disponible en https://www.midagri.gob.pe/portal/download/pd f/ais-2015/relieve-suelo-tierra.pdf

- FAO. [Organización de las Naciones Unidas para la Agricultura y la Alimentación]. (2006). Tendencias y perspectivas del sector forestal en
América latina y el Caribe. Roma. Estudio FAO Montes: 148

- FAO \& SERFOR. (2017). Nuestros bosques en números. Primer reporte del Inventario Nacional Forestal y de Fauna Silvestre. Lima. 1 era edición, junio de 2017 Hecho el Depósito Legal en la Biblioteca Nacional del Perú No 201706574

- INRENA. [Instituto Nacional de Recursos Naturales]. (1997). Compendio estadístico de la actividad forestal del Perú 1980-1996. Disponible en http://sniffs.serfor.gob.pe/estadistica/es/tableros /publicaciones/anuarios

- INRENA. [Instituto Nacional de Recursos Naturales]. (2003). Perú Forestal en números Año 2003. Disponible en http://sniffs.serfor.gob.pe/estadistica/es/tableros /publicaciones/anuarios

- MEF. [Ministerio de Economía y Finanzas]. (2020). Seguimiento de la ejecución presupuestal (consulta amigable). Disponible en https://www.mef.gob.pe/es/seguimiento-de-laejecucion-presupuestal-consulta-amigable

- MINAGRI. [Ministerio de Agricultura y Riego]. (2013). Aprueban la Política Nacional Forestal y de Fauna Silvestre. Decreto Supremo No 0092013-MINAGRI. En: Diario oficial El Peruano: Lima, 14 de agosto del 2013. Año XXX - N 12554, pp: 501081-501089.

- MINAGRI. [Ministerio de Agricultura y Riego]. (2014). Perú Forestal en números 2013. Disponible en http://sniffs.serfor.gob.pe/estadistica/es/tableros /publicaciones/anuarios

- MINAGRI. [Ministerio de Agricultura y Riego]. (2015). Perú Forestal en números 2014. Disponible en http://sniffs.serfor.gob.pe/estadistica/es/tableros /publicaciones/anuarios

- MINAM. [Ministerio del Ambiente]. (2020). $\mathrm{ABC}$ de los bosques peruanos Disponible en http://www.bosques.gob.pe/peru-pais-debosques

- Ramírez, R. (2010). Análisis del modelo de aprovechamiento forestal y fauna silvestre del Perú y propuesta alternativa. Tesis de Doctor, 
Universidad Nacional Federico Villareal, Lima. Perú. 189 pp.

- SERFOR. [Servicio Nacional Forestal y de Fauna Silvestre]. (2016). Anuario Forestal 2015. Disponible

en: http://sniffs.serfor.gob.pe/estadistica/es/tableros /publicaciones/anuarios

- SERFOR. [Servicio Nacional Forestal y de Fauna Silvestre]. (2018). Anuario Forestal y de Fauna Silvestre. (2016). Disponible en: http://sniffs.serfor.gob.pe/estadistica/es/tableros /publicaciones/anuarios
- SERFOR. [Servicio Nacional Forestal y de Fauna Silvestre]. 2019. Anuario Forestal y de Fauna Silvestre. (2017). Disponible en: http://sniffs.serfor.gob.pe/estadistica/es/tableros /publicaciones/anuarios

- SERNANP. [Servicio Nacional de Áreas Naturales Protegidas por el Estado]. (2020). Listado oficial de áreas naturales protegidas actualizado al 27 de febrero del 2020 . Disponible en: https://www.sernanp.gob.pe/documents/10181/ 165150/Listado+ANP+27.02.2020.pdf/f510eb6 a-028c-4d4d-9bad-e43a663cc8c4 